HELSINKI

March 1998

\title{
Spontaneous Discrete Symmetry Breaking During Inflation and the NMSSM Domain Wall Problem
}

\author{
John McDonald 円 \\ Dept. of Physics, \\ P.O.Box 9, \\ University of Helsinki, \\ FIN-00014 Helsinki, \\ FINLAND
}

${ }^{1}$ e-mail: mcdonald@phcu.helsinki.fi 


\begin{abstract}
The Next to Minimal Supersymmetric Standard Model (NMSSM), proposed as a solution of the $\mu$ problem of the Minimal Supersymmetric Standard Model, has a discrete $Z_{3}$ symmetry which is spontaneously broken at the electroweak phase transition, resulting in a cosmological domain wall problem. In most cases this domain wall problem cannot be solved by explicit $Z_{3}$ breaking without introducing supergravity tadpole corrections which destabilize the weak scale hierarchy. Here we consider the possibility of solving the domain wall problem of the NMSSM via spontaneous discrete symmetry breaking occuring during inflation. For the case where the discrete symmetry breaking field has renormalizible couplings to the NMSSM fields, we find that the couplings must be less than $10^{-5}$ if the reheating temperature is larger than $10^{7} \mathrm{GeV}$, but can be up to $10^{-3}$ for reheating temperatures of the order of the electroweak phase transition temperature. For the case of non-renormalizible couplings, we present a model which can solve the domain wall problem for large reheating temperatures without requiring any very small coupling constants. In this model the domain walls are eliminated by a pressure coming from their interaction with a coherently oscillating scalar field whose phase is fixed during inflation. This oscillating scalar field typically decays after the electroweak phase transition but before nucleosynthesis, leaving no additional $Z_{3}$ symmetry breaking in the zerotemperature theory.
\end{abstract}




\section{Introduction.}

The Minimal Supersymmetric extension of the Standard Model (MSSM) has become widely regarded as the most probable theory of physics beyond the Standard Model (SM), due to its ability to protect the weak scale from quadratically divergent radiative corections [酉]. However, the MSSM requires, in order to give an expectation value to both Higgs doublets, the introduction of a SUSY mass parameter for the Higgs doublets, $\mu$, which has no obvious reason to be of the order of $m_{W}$, although some mechanisms have been suggested in the context of supergravity models [2]. (We will refer to this as the $\mu$ problem). One particularly simple and attractive solution of the $\mu$ problem is to extend the MSSM to the $Z_{3}$ symmetric Next to Minimal Supersymmetric Standard Model (NMSSM) [1, 3, 4. This adds a scalar superfield $N$ to the MSSM whose expectation value provides an effective $\mu$ term for the Higgs doublets. The $\mu$ term is naturally of the order of the weak scale so long as the $Z_{3}$ symmetry is imposed. However, the $Z_{3}$ symmetry introduces a cosmological problem in the case where the reheating temperature after inflation is large compared with the weak scale, namely the formation of stable domain walls

[5, 6] due to sponanteous breaking of the $Z_{3}$ symmetry at the electroweak phase transition [7].

In order to eliminate these domain walls, a $Z_{3}$ symmetry breaking which has the same phase over the region corresponding to the observable Universe must be introduced into the effective weak scale theory. However, any explicit $Z_{3}$ symmetry breaking (even if due to Planck-scale suppressed non-renormalizible operators) will almost certainly destabilize the weak scale by allowing quadratically divergent gauge singlet tadpole diagrams which appear in the full supergravity theory [7, 8]. (However, for the special case where the $Z_{3}$ is embedded in a gauged R-symmetry, such corrections can be avoided [9]).

The obvious alternative is to consider spontaneous $Z_{3}$ symmetry breaking due to a new gauge singlet scalar field, $S$, whose initial value is fixed during inflation. This ensures that the resulting discrete symmetry breaking phase is the same over the 
whole observable Universe. In this paper we will discuss whether such a possibility can be implemented in a natural way, in the sense of not requiring extremely small coupling constants. We will consider two different approaches. One approach is based on coupling $S$ directly to the NMSSM via renormalizible couplings. The second is based on having only non-renormalizible couplings of $S$ to the NMSSM sector, suppressed by powers of the Planck scale or some intermediate mass scale.

We first outline the cosmological scenario we have in mind. We will consider the simplest scenario, in which there is an initial period of inflation due to the energy density of an inflaton field, which subsequently oscillates coherently about the minimum of its potential until it decays, leaving the Universe radiation dominated at a reheating temperature $T_{R}$ [10]. $T_{R}$ should be low enough not to thermally regenerate gravitinos [11, [12] $\left(T_{R} \curvearrowright 10^{8}-10^{9} \mathrm{GeV}\right.$ for gravitino masses in the range $100 \mathrm{GeV}$ to $500 \mathrm{GeV}$ [13]), implying that the value of the expansion rate of the Universe at $T_{R}, H\left(T_{R}\right)$, should be less than $1 \mathrm{GeV}$. Between the end of inflation and the beginning of radiation domination the Universe is dominated by the energy density of the coherent inflaton oscillations. An important point to note is that the Universe does not "reheat" as such. During the inflaton oscillation dominated period the decay of the inflaton results in a themal background of particles with a temperature $T_{r} \approx k_{r}\left(M_{P l} H T_{R}^{2}\right)^{1 / 4}$ (where $k_{r} \equiv\left(\frac{9}{5 \pi^{3}} \frac{g\left(T_{R}\right)}{g(T)^{2}}\right)^{1 / 8}$ is approximately equal to 0.4 for temperatures larger than $100 \mathrm{GeV}$ ), the energy density of which eventually comes to dominate the energy density of the Universe at $T_{R}$ [10]. The magnitude of the density perturbations observed by the Cosmic Background Explorer implies that $H \approx 10^{14} \mathrm{GeV}$ during inflation, assuming that the density perturbations are due to quantum fluctuations [14]. Therefore it is possible that $T_{r}$ could exceed $10^{9} \mathrm{GeV}$ at the end of inflation, even if $T_{R}$ is less than $10^{9} \mathrm{GeV}$. In general $T_{r}$ is given by

$$
T_{r} \approx 6.3 \times 10^{9} k_{r}\left(\frac{H}{100 G e V}\right)^{1 / 4}\left(\frac{T_{R}}{10^{9} G e V}\right)^{1 / 2} G e V
$$

Although it might seem that $T_{r} \gtrsim 10^{9} \mathrm{GeV}$ would require a tighter bound on $T_{R}$ in order to evade the thermal regeneration of gravitinos, it is straightforward to show that the rapid increase of $H$ as a function of $T$ during the inflaton oscillation 
dominated period, $H \propto T^{4}$, ensures that the gravitinos generated thermally during this period never exceed those generated around $T_{R}$. Thus it is sufficient to impose $T_{R} \lesssim 10^{9} \mathrm{GeV}$. On the other hand, we will find that the higher temperatures which exist during the inflaton oscillation dominated period must be taken into account when considering constraints on the couplings following from the requirement that the $Z_{3}$ symmetry is not thermally restored before the NMSSM domain walls form.

It has become clear that in many inflation models based on supergravity, the scalar fields will obtain order $H^{2}$ SUSY breaking mass squared terms as a result of the non-zero energy density which exists in the early Universe [10, 15]. (A possible exception is the case of D-term inflation, depending on the details of the model [16]). If such mass squared terms happen to be negative, then any scalar field with a flat scalar potential will have a large initial expectation value which is fixed during inflation. This is in contrast with the case of potentials having hidden sector SUSY breaking mass squared terms, which have a fixed value, $m_{s}^{2}$, which is typically of the order of $(100 \mathrm{GeV})^{2}$. (By "hidden sector" mass terms we mean those coming from a supergravity hidden sector breaking mechanism [1]). In this case the fields cannot roll to the minimum of their potentials during inflation since $H$ is much larger than $m_{s}$ and the fields are overdamped. Therefore they will have random initial values. The role of the $H^{2}$ mass terms in our discussion will be to fix the initial values of the fields at the end of inflation to be at a non-zero minimum of the scalar potential, with a single discrete symmetry breaking phase througout the observed region of the Universe. It is interesting to ask whether the domain wall problem can be solved with random initial values of the fields at the end of inflation, as in the case without the order $H^{2}$ mass terms, but we will not address this question here.

There are then two possible scenarios for the cosmological evolution of the $S$ expectation value, depending on the sign of $m_{s}^{2}$. If this is negative, the $S$ expectation value will remain large and constant once the hidden sector mass squared term comes to dominate the order $H^{2}$ term during the expansion of the Universe. On the other hand, if it is positive, the $S$ scalar will begin to oscillate coherently once 
$m_{s}^{2}$ dominates the $H^{2}$ term. If these $Z_{3}$ symmetry breaking expectation values can survive down to temperatures less than that of the electroweak phase transition, $T_{e w}$, (at which the $Z_{3}$ domain walls form), then we may be able to use the interaction of $<S>$ with the NMSSM sector fields to eliminate the domain walls.

However, we will see that it is not so easy to achieve a natural solution of the domain wall problem. On the one hand, we have to provide a large enough vacuum energy density splitting between the different $Z_{3}$ domains to provide sufficient pressure to drive away the domain walls. On the other hand, for the case of the negative hidden sector mass squared term, we have to ensure that the $Z_{3}$ symmetry is not thermally restored while at the same time ensuring that the $S$ expectation value does not give a large mass to the scalars in the NMSSM sector responsible for electroweak symmetry breaking. For the case of the coherently oscillating $S$ scalar, we have to ensure that the $S$ field is not thermalized before the electroweak phase transition and that it decays after the electroweak phase transition but not so late that it disrupts the predictions of standard big-bang nucleosynthesis [13. 


\section{Renormalizible $S$ couplings to the NMSSM sector.}

(a) $m_{s}^{2}<0$.

We define the transformation of a field $\phi$ with charge $Q(\phi)$ under a discrete symmetry by $\phi \rightarrow e^{i 2 \pi Q} \phi\left(-\frac{1}{2}<Q \leq \frac{1}{2}\right)$. The simplest extension of the NMSSM is then obtained by considering the $Z_{3}$ charge of $S$ to be equal to that of $N, Q(S)=$ $1 / 3$. The superpotential of the model is given by $W=W_{N M S S M}+W_{S}$, where

$$
W_{N M S S M}=W_{\text {Yukawa }}+\lambda_{N} N H_{1} H_{2}-\frac{k}{3} N^{3}
$$

is the standard $Z_{3}$ symmetric NMSSM superpotential [1, 3, 4, 7] and

$$
W_{S}=\lambda_{S H} S H_{1} H_{2}+\frac{\lambda_{1}}{3} S^{3}+\lambda_{2} S^{2} N+\lambda_{3} S N^{2}
$$

gives the couplings of $S$ to the NMSSM fields. In addition we will have soft SUSY breaking terms of the form [15]

$$
\left(m_{s}^{2} \pm H^{2}\right)\left|\phi_{i}\right|^{2}+\left(A_{\alpha} W_{\alpha}+\text { h.c. }\right)
$$

where $\phi_{i}$ are the scalar fields and $W_{\alpha}$ are the trilinear superpotential terms. $\left(A_{\alpha} \sim\right.$ $m_{s} \pm H$ in the presence of non-zero $H$, with the sign of the $H$ correction being model dependent [15]). With these superpotential and soft SUSY breaking terms, the $S$ scalar potential is assumed to be given by

$$
V(S)=\left(-m_{s}^{2}-H^{2}\right)|S|^{2}+\left(A_{\lambda_{1}} \frac{\lambda_{1}}{3} S^{3}+\text { h.c. }\right)+\left(\lambda_{1}^{2}+\lambda_{2}^{2}\right)|S|^{4}
$$

The expectation value of $S$ is then given by

$$
<S>\approx \frac{\left(m_{s}^{2}+H^{2}\right)^{1 / 2}}{\left(\lambda_{1}^{2}+\lambda_{2}^{2}\right)^{1 / 2}}
$$

(We have neglected the $A_{\lambda_{1}}$ term, which can alter $<S>$ by at most factor of the order of 1).

In this we have ignored the $N$ expectation value. In fact, a non zero $\langle N\rangle$ will generally exist at the minimum together with $\langle S\rangle$. This could result in a large effective $\mu$ term and affect the natural generation of the weak scale. We will discuss $N$ expectation value and its consequences later in this section. 
We first consider the conditions under which there is no thermal restoration of the $Z_{3}$ symmetry. This requires that the $S^{2}$ term in the finite temperature effective potential [12, 17] remains negative for all values of $T$ for which the $S$ condensate scalars are in thermal equilibrium.

The $N$ particles and Higgs particles $H_{i}$ will contribute to the $S$ finite-temperature effective potential if they are lighter than $T$. In fact, they will be lighter than $T$ for $T$ large compared with $T_{e w}$, since any $N$ or $H_{i}$ mass term due to $\langle S>$ cannot be very large compared with the weak scale without disrupting electroweak symmetry breaking [4]. The $S$ particles orthogonal to $\langle S\rangle$, which we denote by $S^{\prime}$, will also gain a mass from $\langle S\rangle$ and can contribute to the finite temperature effective potential. The condition for these particles to be light compared with $T$ for $H \underset{\sim}{\sim} m_{s}$ is that

$$
\frac{\lambda_{1}}{\left(\lambda_{1}^{2}+\lambda_{2}^{2}\right)^{1 / 2}} \stackrel{<}{\sim} \frac{T}{m_{s}}(7),
$$

which will generally be satisfied for $T \gtrsim T_{\text {ew }}$. (We need not consider the constraints for $H \gtrsim m_{s}$, since in this case the soft SUSY breaking mass squared in the scalar potential becomes of the order of $H^{2}$, which will increase with $T$ much faster then the thermal $T^{2}$ correction, thus ensuring that the $Z_{3}$ symmetry remains broken at $H \gtrsim m_{s}$ if it is broken for all $\left.H \lesssim m_{s}\right)$. The effective SUSY $N$ mass from the $\lambda_{3}$ coupling is then given by $m_{N}(S)=2 \lambda_{3} S$. Similar contributions will come from $\lambda_{1}$ and $\lambda_{2}$.

We must then ensure that the temperature dependent contribution to the $S^{2}$ term in the finite temperature effective potential due to $m_{N}, m_{N}^{2}(S) T^{2} / 8$, is small compared with $m_{s}^{2} S^{2}$ for all $T \lesssim T_{e q}$, where $T_{e q}$ is the temperature at which the $S$ scalars come into thermal equilibrium. The dominant process bringing the $S$ scalars into thermal equilibrium with the light particles in the thermal background will be inverse decays, with a rate $\Gamma_{i n v} \approx \frac{\kappa_{d} \lambda_{i}^{2}}{4 \pi} T$ (where the factor $\kappa_{d}$ is not too small compared with 1 ; for the case of stop quarks this has been estimated to be typically of the order of 0.1 [18]). If $T_{e q} \lesssim T_{R}$, then the thermal equilibrium condition $\Gamma_{i n v} \gtrsim H$ 
occurs during radiation domination and $T_{e q}$ is given by

$$
T_{e q} \approx \frac{\kappa_{d} \lambda_{i}^{2} M_{P l}}{4 \pi k_{T}}
$$

where the expansion rate during radiation domination is given by $H=\frac{k_{T}(T) T^{2}}{M_{P l}}$, with $k_{T}(T) \approx 16$ for $T$ larger than around $100 \mathrm{GeV}$ [12]. This is consistent with $T_{e q} \lesssim T_{R}$ if

$$
\lambda_{i} \lesssim 9 \times 10^{-6}\left(\frac{4 \pi k_{T}}{\kappa_{d}}\right)^{1 / 2}\left(\frac{T_{R}}{10^{9} G e V}\right)^{1 / 2}
$$

If this is not satisfied then $T_{e q} \gtrsim T_{R}$ and, with $H(T)$ given by $H(T)=T^{4}\left(k_{r}^{4} T_{R}^{2} M_{P l}\right)^{-1}$, the thermal equilibrium temperature becomes

$$
T_{e q} \approx\left(\frac{\kappa_{d} k_{r}^{4}}{4 \pi}\right)^{1 / 3} \lambda_{i}^{2 / 3} T_{R}^{2 / 3} M_{P l}^{1 / 3}
$$

The $Z_{3}$ symmetry remains broken at $T_{e q}$ if $\lambda_{i} \lesssim m_{s} / T_{e q}$. For $T_{e q} \lesssim T_{R}$ this requires that

$$
\lambda_{i} \propto\left(\frac{4 \pi k_{T}}{\kappa_{d}}\right)^{1 / 3}\left(\frac{m_{s}}{M_{P l}}\right)^{1 / 3} \approx 1.3 \times 10^{-5} \kappa_{d}^{-1 / 3}\left(\frac{m_{s}}{100 G e V}\right)^{1 / 3}
$$

where we have used $k_{T} \approx 16$. This is the condition for avoiding $Z_{3}$ symmetry restoration if $T_{R} \gtrsim 10^{7} \mathrm{GeV}$. If $T_{R}$ is less than $10^{7} \mathrm{GeV}$, the upper limit on $\lambda_{i}$ occurs when $T_{e q}>T_{R}$ and the condition that the $Z_{3}$ symmetry remains broken at $T_{e q}$ becomes

$$
\lambda_{i} \lesssim 2.1 \times 10^{-6} \kappa_{d}^{-1 / 5}\left(\frac{10^{9} G e V}{T_{R}}\right)^{2 / 5}\left(\frac{m_{s}}{100 G e V}\right)^{3 / 5}
$$

For $T_{R}=10^{2} \mathrm{GeV}\left(10^{5} \mathrm{GeV}\right)$ this requires that $\lambda_{i} \lesssim 10^{-3}\left(10^{-4}\right)$. Thus we see that, for the case of $m_{s}^{2}<0$ and renormalizible $S$ couplings to the NMSSM sector, the couplings of $S$ to $N$ and to the Higgs fields must be rather small if the reheating temperature is large compared with $T_{e w}$, in order not to thermally restore the $Z_{3}$ symmetry. In particular, if the reheating temperature is close to the gravitino upper limit, then these couplings must be less than around $10^{-5}$.

We have not yet discussed the elimination of the domain walls. We will discuss this in more detail later, but for now we merely note that it is very easy to introduce 
large $<S>$ dependent contributions into the $N$ scalar potential. For example, from the superpotential couplings $k$ and $\lambda_{2}$, we obtain a term in the scalar potential

$$
-2 \lambda_{2} k<S^{\dagger}>^{2} N^{2}+\text { h.c. } \sim\left(\frac{2 \lambda_{2}}{\lambda_{1}^{2}+\lambda_{2}^{2}}\right) k m_{s}^{2}\left(N^{2}+\text { h.c. }\right)
$$

However, if we assume that $k$ is not very small compared with 1 , such that $2 k>\lambda_{2}$, then $\lambda_{2}$ must be smaller than $\lambda_{1}^{2} / k$ in order to avoid introducing a mass term for the $N$ scalars much larger than $10^{2} \mathrm{GeV}$. In the NMSSM broken by radiative corrections, the phenomenologically favoured region of parameter space has $\langle N\rangle \gtrsim 1 T e V$. In this case the $N$ particles essentially decouple and one has an effective MSSM with $\mu \equiv \lambda_{N}<N>$ and a contribution to the soft SUSY breaking " $B$-term" given by $\nu=k<N>\llbracket$. Both of these cannot be very much larger than $10^{2} \mathrm{GeV}$ without disrupting the natural generation of the weak scale. (It is possible to have very large $\langle N\rangle$, so long as $\lambda_{N}$ and $k$ are correspondingly small. The condition for a weak scale $\mu$ term is then that $\lambda_{N} / k \sim 1$ [ [4] $)$. The effect of a large mass term from equation (13) would be to introduce an effective negative mass squared, $m_{N \text { eff }}^{2}$, for the $N$ scalars, with the $N$ expectation being given by $\left\langle N>\approx\left|m_{N \text { eff }}\right| / k\right.$, which would result in $\nu \approx\left|m_{N \text { eff }}\right|$ [4]. Requiring that $\nu$ be small compared with a mass scale $m_{*}$ not much larger than $10^{2} \mathrm{GeV}$ and that the no-thermalization condition (12) is satisfied then implies that

$$
\lambda_{2} \lesssim 2.2 \times 10^{-10} \kappa_{d}^{-2 / 5}\left(\frac{0.01}{k}\right)\left(\frac{10^{9} G e V}{T_{R}}\right)^{4 / 5}\left(\frac{m_{s}}{100 G e V}\right)^{-4 / 5}\left(\frac{m_{*}}{100 G e V}\right)^{2}
$$

(For $T_{R} \gtrsim 10^{7} \mathrm{GeV}$ the upper bound from (14) with $T_{R} \approx 10^{7} \mathrm{GeV}$ applies). Thus for large reheating temperatures, $T_{R} \gtrsim 10^{7} \mathrm{GeV}$, we would require that $\lambda_{2} \lesssim 10^{-7}(0.01 / k)$ in order to avoid a mass term for the $N$ scalars much larger than $10^{2} \mathrm{GeV}$. For smaller $T_{R}$ this upper bound becomes weaker, allowing $\lambda_{2}$ to be as large as $10^{-3}(0.01 / k)$

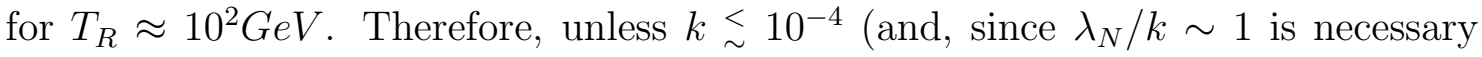
[由], $\lambda_{N} \curvearrowright 10^{-4}$ ), for large $T_{R}$ there will be a tighter upper bound on some of the couplings than that coming from $Z_{3}$ symmetry restoration.

We next consider the $N$ expectation value. When $\langle S\rangle$ is introduced into the $N-S$ scalar potential, terms linear, quadratic and cubic in $N$ arise, which result 
in a non-zero $\langle N>$. In general it is difficult to discuss the minimization of the potential analytically, but for the natural case where the couplings $\lambda_{i}(\mathrm{i}=1,2,3)$ all have the same magnitude and $|k|$ is large compared with $\left|\lambda_{i}\right|$, and where $\langle N\rangle$ does not alter the value of $\langle S\rangle$ significantly, we find that $\langle N\rangle$ is essentially determined by the linear and quartic terms in the $N$ scalar potential. In this case $<N>$ is given by

$$
<N>\approx\left(\frac{\lambda_{i}}{k}\right)^{2 / 3}<S>
$$

(This will not affect the value of $\langle S\rangle$ so long as $\langle N\rangle$ is small compared with $<S>$ ). The main effect of $\langle N\rangle$ will be to introduce an effective $\mu$ term for the Higgs doublets via the $\lambda_{N}$ superpotential coupling,

$$
\mu \approx\left(\frac{k}{\lambda_{i}}\right)^{1 / 3}\left(\frac{\lambda_{N}}{k}\right) m_{s} \quad(16) .
$$

Since $\lambda_{N}$ and $k$ must be of the same order of magnitude to naturally generate a weak scale $\mu$ term, we see that $\lambda_{i}$ cannot be too small compared with $k$, thus favouring large $\lambda_{i}$ and/or small $k$. We note that $\langle S\rangle$ also introduces a $\mu$ term via the $\lambda_{S H}$ superpotential coupling. This will be acceptable so long as $\lambda_{S H}$ is not larger than $\left(\lambda_{1}^{2}+\lambda_{2}^{2}\right)^{1 / 2}$.

We should check that spontaneous breaking of the discrete symmetry does not introduce dangerous quadratic divergences. A quadratically divergent tadpole arises at two-loops in $N=1$ supergravity with hidden sector SUSY breaking and no direct coupling of the hidden and observable sectors in the Kahler potential [8]. With a Kahler potential given by

$$
K=N^{\dagger} N+H_{i}^{\dagger} H_{i}+O\left(\frac{1}{M_{P l}^{2}}\right)
$$

corresponding to minimal scalar kinetic terms, and superpotential terms of the form [8]

$$
W=\ldots+\lambda N H_{1} H_{2}-\frac{\lambda_{\alpha}}{M_{P l}} N^{2} H_{1} H_{2}+O\left(\frac{1}{M_{P l}^{2}}\right)
$$

a divergent $N$ tadpole is generated

$$
\frac{2 \lambda \lambda_{\alpha} \Lambda^{2}}{\left(16 \pi^{2}\right)^{2}} \int d^{4} \theta e^{K / M_{P l}^{2}}\left(\frac{N+N^{\dagger}}{M_{P l}}\right)
$$


On introducing the hidden sector SUSY breaking F-term for $K, F_{K}^{2} \theta^{2} \bar{\theta}^{2}$ with $F_{K}=$ $\left(\frac{m_{s} M_{P l}}{\sqrt{8 \pi}}\right)$, there is a contribution to the $N$ scalar potential of the form 8

$$
\frac{2 \lambda \lambda_{\alpha} \Lambda^{2}}{\left(16 \pi^{2}\right)^{2} M_{P l}^{3}}\left(\frac{m_{s} M_{P l}}{\sqrt{8 \pi}}\right)^{2}\left(N+N^{\dagger}\right) \quad(20) .
$$

Taking the cut-off $\Lambda$ to be $M_{P l}$, we find that the additional term in the $N$ scalar potential is of the form $\bar{M}^{3}\left(N+N^{\dagger}\right)$, where $\bar{M} \approx 7 \times 10^{5} \lambda^{1 / 3} \lambda_{\alpha}^{1 / 3} \mathrm{GeV}$. If $\bar{M}$ is much larger than $10^{2} \mathrm{GeV}$, this term will make impossible the generation of a naturally weak scale $\mu$ term. Throughout this paper we will consider the natural value of non-renormalizible couplings such as $\lambda_{\alpha}$ to be of the order of 1, with the strength of the couplings set by the large mass scale which we will take to be the Planck scale. In this case we would require that $\lambda \lesssim 10^{-12}$ in order to have $\bar{M} \lesssim 100 \mathrm{GeV}$. In the case where discrete symmetry breaking is spontaneous and non-vanishing at zero temperature, we can still have quadratically divergent tadpoles. For example, for $S$ with charge $Q(S)=1 / 3$, one can have a non-renormalizible operator of the form of the $\lambda_{\alpha}$ term but with an overall factor of $\left(\frac{<S>}{M_{P l}}\right)^{2}$. For $S$ with charge $Q(S)=1 / 6$ this factor becomes $\left(\frac{<S>}{M_{P l}}\right)^{4}$. As a result, the quadratically divergence is not a problem for all $\lambda \lesssim 1$ if $\left\langle S>\approx 10^{13} \mathrm{GeV}\right.$ for the $Q(S)=1 / 3$ case and $<S>\lesssim 10^{16} \mathrm{GeV}$ for the $Q(S)=1 / 6$ case. From equation (6) we see that, for $m_{s} \approx 10^{2} \mathrm{GeV},\left\langle S>\lesssim 10^{13} \mathrm{GeV}\right.$ requires that $\left(\lambda_{1}^{2}+\lambda_{2}^{2}\right)^{1 / 2} \gtrsim 10^{-11}$. This can be easily satisfied. 
(b) $m_{S}^{2}>0$.

In this case we will have a coherently oscillating $S$ scalar. We have to ensure that the $Z_{3}$-breaking condensate does not thermalize or decay before the electroweak phase transition.

The requirement that the condensate does not thermalize via inverse decays imposes the constraint,

$$
\lambda_{i} \stackrel{\sim}{\sim}\left(\frac{4 \pi k_{T}}{\kappa_{d}}\right)^{1 / 2}\left(\frac{T}{M_{P l}}\right)^{1 / 2}
$$

where we have assumed that the Universe is radiation dominated. Thus, with $T \approx$ $T_{e w}$, this requires that $\lambda_{i} \lesssim 10^{-8}$. (Below $T_{e w}$ the $S^{\prime}, H_{i}$ and $N$ particles gain masses from electroweak symmetry breaking). This constraint should be applied if the masses of the $S^{\prime}, H_{i}$ and $N$ particles due to $\langle S>$ are small compared with T. During the inflaton oscillation dominated era, the energy density of the $S$ oscillations scales as that in the inflaton oscillations, whilst after reheating the energy density of $S$ oscillations scales as $a(T)^{-3}$, where $a(T)$ is the scale factor. Thus the energy density during radiation domination at $T<T_{R}$ is given by

$$
\rho_{S}(T)=\frac{\alpha_{\rho}(T) T^{3} T_{R}}{m_{s}^{2} M_{P l}^{2}} \rho_{o} \quad(22),
$$

where $\rho_{o}$ is the initial energy density of the $S$ condensate and $\alpha_{\rho}(T)=\frac{g(T)}{g\left(T_{R}\right)} k_{T}\left(T_{R}\right)^{2}$. The the corresponding amplitude of the $S$ oscillations at temperature $T$ is $S(T) \approx$ $\left(\rho_{S} / \rho_{o}\right)^{1 / 2} S_{o}$, where $S_{o}$ is the initial amplitude of the oscillations. The condition that the mass of the particles due to their interaction with the condensate [19], $\lambda_{i} S(T)$, is less than $T$ then becomes

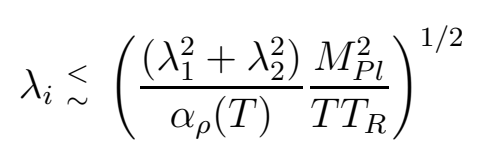

This will be easily satisfied for $T_{e w} \lesssim T \lesssim T_{R}$. Therefore (21) applies. We should also check that the energy density is not dominated by the coherent $S$ oscillations. This is satisfied if $\lambda_{1}^{2}+\lambda_{2}^{2} \gtrsim 10^{-28} m_{s}^{2} / T$, which is typically easily satisfied.

Under the assumption that the $S$ condensate particles are heavy enough to decay directly to a two-body final state via the coupling $\lambda_{i}$, with a rate $\Gamma_{d} \approx \frac{\lambda_{i}^{2}}{4 \pi} m_{s}$, the 
requirement that the condensate does not decay before temperature $T$ imposes the constraint

$$
\lambda_{i} \lesssim\left(4 \pi k_{T}\right)^{1 / 2}\left(\frac{T^{2}}{m_{s} M_{P l}}\right)^{1 / 2}
$$

This will give an even tighter constraint on $\lambda_{i}$ than the no-thermalization constraint (21) if the coherent oscillations have to survive down to a temperature much lower than the electroweak phase transition in order to eliminate the domain walls, as is likely to be the case in the NMSSM [0].

We next consider the conditions under which the $S$ condensate can eliminate the NMSSM domain walls. This requires that the pressure due to the energy difference $\Delta V$ between the domains is large enough to cause the higher energy domains to collapse on the time scale $H(T)^{-1}$ [5, 6, 20]. The condition on $\Delta V$ depends on whether the domain walls are relativistic or not, i.e. whether there are significant frictional forces due to the interaction of the domain walls with the thermal background particles. If there are strong frictional forces, then the walls will expand until the force due to surface tension is balanced by the friction, which will occur at a radius much less than the horizon $H^{-1}(T)$. The pressure due to $\Delta V$ must then overcome this large frictional force. If there are effectively no frictional forces, meaning that the surface tension can overcome the frictional forces for relativistic walls of radius equal to the horizon, then the condition on $\Delta V$ will be typically much weaker. For the case of an unbroken $Z_{3}$ symmetry, the conventional view would be that there are no (or at least highly suppressed) frictional forces, since the particles crossing the domain walls would have equal masses on either side of the wall and so would transfer no net momentum to the wall on passing through [20, 21]. However, it has been suggested that, on taking into account the change in the particle masses in the vicinity of the wall, there will be a non-zero reflection coefficient, leading to a large frictional force until the heavier particles become Boltzmann suppressed at temperatures less than around that of the quark-hadron phase transition, after which the domain walls become relativistic on the scale of the horizon [7]. In more detail, the friction force per unit area on the wall is estimated to be $f \approx \frac{g}{8 \pi^{2}} \frac{m^{5}}{m_{W}^{2}} v T$, 
where $m$ is the mass of the heaviest thermal background particle with $m<T, g$ is the number of degrees of freedom of the particle and $v$ is the velocity of the wall [0]. This force rapidly decreases as $T$ decreases and the heavier thermal particles become Boltzmann suppressed. The condition on $\Delta V$ to eliminate the domain walls, $\Delta V \gtrsim f$, will be most easily satisfied once the frictional force is weak enough to allow the domain walls to remain relativistic up to scales of the order of the horizon. Thereafter, the condition will become more difficult to satisfy as $T$ decreases, since the pressure difference between the domains rapidly decreases as $\langle S>$ decreases with $T$.

Suppose the domain walls become relativistic on horizon scales at $T_{\text {rel }}$. Soon after $T_{r e l}$, the uncollapsed domain walls will have a radius of the order of the horizon $H\left(T_{r e l}\right)^{-1}$. This will continue to be true until the pressure due to $\Delta V$ dominates the force due to the surface tension $\sigma$. This in general occurs once the radius of a domain is larger than a critical radius, $r_{c}$, given by

$$
r_{c} \approx \frac{\sigma}{|\Delta V|}(25)
$$

The smallest $|\Delta V|$ will therefore correspond to the largest possible $r_{c}$, the horizon radius. Although the $Z_{3}$ domain wall will consist of the Standard Model Higgs fields as well as the $N$ field, for simplicity we will consider the wall to be made only of the $N$ field. In this case the domain wall potential will be due to the soft SUSY breaking $N^{3}$ A-term and the surface tension will be $\sigma \approx m_{s}<N>^{2}$ [6, 20].

From the superpotential couplings $\lambda_{2}$ and $k$ we obtain an energy density splitting between the different $Z_{3}$ vacuum states

$$
\Delta V \approx 2 \lambda_{2} k S^{2} N^{2} \quad(26)
$$

This depends on the value of $S^{2}$ and so will have a non-zero value on averaging over the $S$ oscillations. The condition that this can overcome the surface tension for horizon-sized relativistic domains at $T_{r e l}$ is then that

$$
\frac{\lambda_{1}^{2}+\lambda_{2}^{2}}{2 k \lambda_{2}} \lesssim \frac{\alpha_{\rho}\left(T_{r e l}\right)}{k_{T}\left(T_{r e l}\right)} \frac{T_{R} T_{r e l}}{m_{s} M_{P l}}
$$


The left-hand side will be smallest for $\lambda_{1} \lesssim \lambda_{2}$, so we require that

$$
\frac{\lambda_{1}}{k} \underset{\sim}{\sim} \frac{\lambda_{2}}{k} \lesssim \frac{10^{-13} \alpha_{\rho}\left(T_{r e l}\right)}{k_{T}\left(T_{r e l}\right)}\left(\frac{T_{R}}{10^{9} G e V}\right)\left(\frac{T_{r e l}}{0.1 G e V}\right)\left(\frac{100 G e V}{m_{s}}\right)
$$

Therefore we see that the requirement that $\Delta V$ is large enough to eliminate the domain walls imposes a very tight constraint on at least some of the couplings. A similar $S^{2}$ dependent contribution to $\Delta V, \Delta V \approx 2 \lambda_{1} \lambda_{3} S^{2} N^{2}$, arises from the superpotential couplings $\lambda_{1}$ and $\lambda_{3}$. For this to eliminate the domain walls we would require that

$$
\frac{\lambda_{2}}{\lambda_{3}} \lesssim \frac{\lambda_{1}}{\lambda_{3}} \lesssim \frac{10^{-13} \alpha_{\rho}\left(T_{r e l}\right)}{k_{T}\left(T_{r e l}\right)}\left(\frac{T_{R}}{10^{9} G e V}\right)\left(\frac{T_{r e l}}{0.1 G e V}\right)\left(\frac{100 G e V}{m_{s}}\right)
$$

which would demand even smaller values of $\lambda_{1}$ and $\lambda_{2}$ once the no-thermalization and decay constraints, (21) and (24), are imposed.

Thus we can conclude that, for the case of renormalizible couplings of the $Z_{3}$ symmetry breaking scalar $S$ to the NMSSM, very small couplings (less than $10^{-8}$ and in some cases less than $10^{-13}$ ) are required in order to eliminate the NMSSM domain walls if the hidden sector mass squared of $S$ is positive. If it is negative, small couplings $\lesssim 10^{-5}$ (and even smaller if the NMSSM couplings $k$ and $\lambda_{N}$ are larger than $10^{-4}$ ) are again required if the reheating temperature is larger than $10^{7} \mathrm{GeV}$, but, for smaller values of $T_{R}$, the constraints from avoiding thermal restoration of the $Z_{3}$ symmetry become somewhat weaker, allowing couplings as large as $10^{-3}$ for $T_{R} \approx T_{e w}$. 


\section{Non-Renormalizible $S$ couplings to the NMSSM Sector.}

We have seen that the case of renormalizible $S$ couplings to the NMSSM sector requires rather small couplings when the reheating temperature is large (less than $10^{-5}$ for the case where the reheating temperature is larger than $10^{7} \mathrm{GeV}$, with an even smaller upper bound if the NMSSM $N$ couplings are not less than about $10^{-4}$ ). In particular, for the case of a positive hidden sector mass squared for the $S$ scalar, some couplings must be less than about $10^{-13}$, regardless of the reheating temperature. One way to overcome the need for such small couplings might be to simply eliminate all the renormalizible couplings of the $S$ scalar to the NMSSM fields, allowing only non-renormalizible couplings suppressed by powers of some large mass scale (which we will take to be the Planck scale). This is naturally achieved by extending the $Z_{3}$ symmetry of the NMSSM to a discrete symmetry, which we will call $Z_{A}$, under which the charge of $S$ is such that no renormalizible couplings of $S$ to the NMSSM fields are possible.

(a) $m_{s}^{2}<0$.

In this case there will be a large and constant expectation value for the $S$ scalar once $H \lesssim m_{s}$. This large expectation value will introduce what we believe to be a generic problem in this case, namely a very large SUSY mass for $N$, much larger than the weak scale. To see this, consider the simplest example, corresponding to $S$ having a $Z_{A}$ charge $1 / 6$ whilst $N$, as usual, has charge $1 / 3$. Then $S$ can only have leading order non-renormalizible superpotential couplings of the form

$$
\frac{\lambda}{M_{P l}} S^{2} N^{2}+\frac{\lambda^{\prime}}{M_{P l}} S^{2} H_{1} H_{2}+\frac{\lambda_{S}}{6 M_{P l}^{3}} S^{6} \quad \text { (30). }
$$

The expectation value following from the $S$ scalar potential,

$$
V(S)=-m_{s}^{2} S^{2}+\left(\frac{\lambda_{S}}{M_{P l}^{3}}\right)^{2} S^{10} \quad(31),
$$

gives, with $m_{s} \approx 10^{2} \mathrm{GeV}$ and $\lambda_{S} \approx 1,<S>\approx 5 \times 10^{14} \mathrm{GeV}$. This results in an SUSY mass for $N$ of the order of $10^{10} \mathrm{GeV}$, making a solution of the $\mu$ problem impossible in this case. We find that decreasing the $Z_{A}$ charge of $S$ or introducing an intermediate mass scale (in the manner of the model to be described in the 
following section) only increases the $N$ mass. Based on this, we conclude that the case of $S$ scalars with non-renormalizible couplings and a negative hidden sector mass squared is unlikely to provide a natural solution of the NMSSM domain wall problem.

(b) $m_{s}^{2}>0$.

So far we have found no model which can solve the NMSSM domain wall problem for reheating temperatures much larger than the electroweak phase transition temperature without requiring small couplings. We now present a model, based on a coherently oscillating scalar, which can eliminate the domain wall problem of the NMSSM for values of $T_{R}$ as large as the gravitino upper bound and which does not require any small couplings.

The model we consider requires, in addition to the NMSSM singlet $N$ and the $Z_{A}$ breaking field $S$, two additional singlet fields, $X$ and $B . X$ will acquire an intermediate mass as a result of an expectation value for $B$. The model is also assumed to have a more complicated discrete symmetry, $Z_{A} \times Z_{B}$, where $Z_{A}$ is the extension of the original NMSSM $Z_{3}$ whilst $Z_{B}$ is a discrete symmetry under which all the NMSSM fields are singlets. $Z_{B}$ is introduced in order to control the nonrenormalizible terms. The discrete symmetry charges of the fields are as defined in Table 1(a).

Table 1(a). Field charges under $Z_{A} \times Z_{B}$.

\begin{tabular}{|c||c|c|c|c|}
\hline & $S$ & $X$ & $B$ & $N$ \\
\hline$Z_{A}$ & $\frac{1}{6}$ & $\frac{1}{2}$ & 0 & $\frac{1}{3}$ \\
$Z_{B}$ & $-\frac{1}{3}$ & $\frac{1}{3}$ & $\frac{1}{6}$ & 0 \\
\hline
\end{tabular}

It may well be significant that the $Z_{A} \times Z_{B}$ charges of the remaining NMSSM fields can always be chosen to eliminate the dangerous renormalizible $B$ and $L$ violating operators $u^{c} d^{c} d^{c}, d^{c} Q L$ and $e^{c} L L$ [1]. For example, this is true for the charges of Table 1(b). Therefore the absence of these renormalizible interactions could be interpreted as a sign of the existence of a non-trivial discrete symmetry such as $Z_{A} \times Z_{B}$, rather than of a simple R-parity or conservation of $\mathrm{B}$ and $\mathrm{L}$. 
Table 1(b). Example of field charges eliminating dangerous renormalizible operators.

\begin{tabular}{|c||c|c|c|c|c|c|c|}
\hline & $e^{c}$ & $L$ & $d^{c}$ & $H_{d}$ & $Q$ & $u^{c}$ & $H_{u}$ \\
\hline$Z_{A}$ & $-\frac{1}{6}$ & $\frac{1}{2}$ & $\frac{1}{3}$ & $-\frac{1}{3}$ & 0 & 0 & 0 \\
$Z_{B}$ & $\frac{1}{3}$ & $-\frac{1}{3}$ & 0 & 0 & 0 & 0 & 0 \\
\hline
\end{tabular}

The superpotential of the model is then given by

$$
W=W_{N M S S M}+W_{X}+W_{N R} \quad(32),
$$

where $W_{X}$ is the allowed renormalizible coupling of $X$,

$$
W_{X}=\lambda_{a} X S N
$$

and $W_{N R}$ gives the leading order non-renormalizible superpotential couplings involving the additional gauge singlet scalars,

$$
W_{N R}=\frac{\lambda_{S}}{6 M_{P l}^{3}} S^{6}+\frac{\lambda_{B X}}{M_{P l}} B^{2} X^{2}+\frac{\lambda_{B}}{6 M_{P l}^{3}} B^{6}
$$

The model will require an intermediate mass for the $X$ scalar. This will be generated by an expectation value for $B$. The obvious possibility is to assume that the hidden sector mass squared of the $B$ scalar is negative, which would result in an expectation value for $B$ given by,

$$
<B>\approx\left(\frac{m_{s}^{2} M_{P l}^{6}}{5 \lambda_{B}^{2}}\right)^{1 / 8} \approx 5 \times 10^{14} \mathrm{GeV}
$$

However, we should perhaps note that in this case there could be problem due to the late decay of oscillations of the weakly coupled $B$ scalar around the minimum of its potential. The $B$ scalar has a mass of the order of $m_{s}$, whilst the $X$ particles to which it couples gain a mass of the order of $10^{10} \mathrm{GeV}$ as a result of $\langle B\rangle$. Thus the $B$ decay rate will be highly suppressed in this case. If the $B$ field is not very close to the minimum of its potential at the end of inflation, its coherent oscillations about the minimum of its potential will decay long after nucleosynthesis and could dominate the energy density of the Universe when they decay. This is 
all dependent upon the value of $B$ at the end of inflation and the dynamics of its subsequent evolution as the Universe expands and its effective (negative) $O\left(H^{2}\right)$ SUSY breaking mass term decreases. Since in our model the $B$ field only serves to generate an intermediate mass for $X$ and, in a sense, may be regarded as a toy field representing the dynamics of a more realistic model, we will not concern ourselves with the details of this issue here.

In general we will consider $M_{X} \gtrsim 10^{10} \mathrm{GeV}$. One reason for this choice, as we will show later, is that it ensures that the $X$ particles are Boltzmann suppressed for all temperatures, thus eliminating the possibility of thermalization of the $S$ condensate by light thermal $X$ particles. Integrating out the massive $\mathrm{X}$ field, we obtain the following effective interaction in the superpotential

$$
\frac{\lambda_{a}^{2}}{M_{X}} S^{2} N^{2}
$$

This is valid so long as $\lambda_{a}\left\langle S>\underset{\sim}{<} M_{X}\right.$, such that the mixing between the $X$ and $N$ fields via the coupling (33) may be neglected. This interaction is fundamentally important in what follows, as it will allow both a sufficiently rapid decay of the $S$ oscillations so as to avoid problems with nucleosynthesis and at the same time allow a sufficiently strong interaction of the $S$ oscillations with the $N$ scalars to drive away the $Z_{3}$ domain walls. Assuming that the $S$ scalar mass is large enough, the $S$ scalars will decay to $N$ particles and $S$ fermions (which will be highly decoupled and will typically have a mass not much larger than $1 \mathrm{eV}$, coming from the interaction (36) with $<N>\sim 100 \mathrm{GeV}-1 \mathrm{TeV})$. So long as their energy density is sufficiently small compared with than that of the radiation when they are produced, the $S$ fermions will not give rise to any cosmological problems. (This may be difficult to achieve, however, if the Universe is dominated by the $S$ scalars when they decay, since one would expect approximately $1 / 3$ of the energy density from $S$ decays to be in the form of $S$ fermions. Alternatively, for the charge assignment of Table 1(b), one can obtain an operator of the same form as equation (36) but with $S^{2} N^{2} \rightarrow S N H_{u} L$, which can allow the $S$ scalars to safely decay to NMSSM fields). 
The $S$ scalar potential is given by

$$
V(S) \approx\left(m_{s}^{2}-H^{2}\right) S^{2}+\left(\frac{\lambda_{S}}{M^{3}} S^{5}\right)^{2}
$$

The initial value of the $S$ field when the oscillations begin at $H \approx m_{s}, S_{o}$, is therefore given by

$$
S_{o} \approx\left(\frac{m_{s}^{2} M_{P l}^{6}}{5 \lambda_{S}^{2}}\right)^{1 / 8}
$$

We first consider the conditions under which the $S$ energy density decays at a temperature less than that of electroweak phase transition, $T_{e w}$, without disturbing the predictions of nucleosynthesis. This requires either that the ratio of the energy density in the $S$ scalars to that in radiation when the $S$ scalars decay, $r_{D}$, is less than $10^{-6}$, in order not to photodissociate the helium abundance [13, 22], or that the $S$ scalars decay at a temperature greater than around $10 \mathrm{MeV}$ [23]. The former condition requires that

$$
T_{R}<\frac{3 r_{D}}{8 \pi} \frac{M_{P l}^{2}}{S_{o}^{2}} T_{d}
$$

The $S$ scalars in the condensate decay via the interaction of equation (36) with a rate given approximately by

$$
\Gamma_{d} \approx \frac{\alpha_{d} \lambda_{a}^{4} m_{S}^{3}}{M_{X}^{2}} \quad(40)
$$

where $\alpha_{d} \approx \frac{1}{192 \pi^{3}} \approx 10^{-4}$ and we are assuming that the $S$ scalars are heavy compared with the $N$ particles. This gives for the decay temperature, assuming that decay occurs during radiation domination,

$$
T_{d} \approx \frac{\alpha_{d}^{1 / 2} \lambda_{a}^{2}}{k_{T}\left(T_{d}\right)^{1 / 2}}\left(\frac{M_{P l} m_{s}^{3}}{M_{X}^{2}}\right)^{1 / 2}
$$

Therefore, in the case where decay occurs after nucleosynthesis at $T_{d} \lesssim 1 \mathrm{MeV}$, we require from equation (39) that the reheating temperature satisfies,

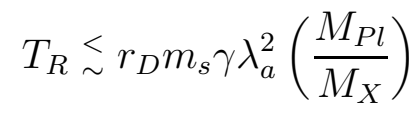

where

$$
\gamma=\frac{3 \alpha_{d}^{1 / 2} \lambda_{s}^{1 / 2}}{8 \pi k_{T}\left(T_{d}\right)^{1 / 2}}
$$


Thus with $r_{D} \approx 10^{-6}, \gamma \approx 10^{-4}, m_{S} \approx 10^{2} \mathrm{GeV}$ and $M_{X} \gtrsim 10^{10} \mathrm{GeV}$, this requires that $T_{R} \lesssim \alpha_{d}^{1 / 2} \lambda_{a}^{2} 10^{4} G e V$. This imposes a severe restriction on $T_{R}$ for $\lambda_{a} \lesssim 0.1$. On the other hand, from equation (41), we see that it is quite likely that $T_{d} \gtrsim 10 \mathrm{MeV}$. In general, $T_{d}$ is given by

$$
T_{d} \approx 10\left(\frac{\alpha_{d}}{10^{-4}}\right)^{1 / 2}\left(\frac{\lambda_{a}}{0.1}\right)^{2}\left(\frac{m_{s}}{100 G e V}\right)^{3 / 2}\left(\frac{10^{10} G e V}{M_{X}}\right) M e V
$$

Therefore, in this case we expect that, for $M_{X} \approx 10^{10} \mathrm{GeV}$ (which will be seen to be the preferred order of magnitude for $M_{X}$ in this model), the $S$ oscillations will typically decay at a temperature between $10 \mathrm{MeV}$ and $1 \mathrm{GeV}$ for $\lambda_{a}$ in the range 0.1 to 1 . The amplitude of the $S$ oscillations at $T<T_{R}$ (assuming that the Universe is radiation dominated) is given by

$$
<S>\approx \frac{16 \alpha_{\rho}(T)^{1 / 2}}{\lambda_{S}^{1 / 4}}\left(\frac{m_{s}}{100 G e V}\right)^{3 / 4}\left(\frac{T}{m_{s}}\right)^{3 / 2}\left(\frac{T_{R}}{10^{9} G e V}\right)^{1 / 2} G e V
$$

The corresponding energy density in the coherent $S$ oscillations is $\rho_{S} \approx m_{s}^{2} S^{2}$. This will come to dominate the radiation energy density once $T \stackrel{\sim}{\sim} T_{\text {dom }}$, where

$$
T_{d o m} \approx \frac{21}{\lambda_{s}^{1 / 2}}\left(\frac{m_{s}}{100 G e V}\right)^{1 / 2}\left(\frac{T_{R}}{10^{9} G e V}\right) G e V
$$

Thus if the domain walls become relativistic below $T_{\text {dom }}$, the elimination of the domain walls by the condensate will occur whilst the Universe is dominated by the energy density of the oscillating $S$ field and possibly whilst the background radiation is dominated by radiation coming from the decays of the condensate scalars. In the case where the $S$ scalars decay during matter domination, it would seem that equation (41), derived assuming radiation domination, is not correct. However, since the decay condition is given by $\Gamma_{d} \approx H \propto \rho_{S}^{1 / 2}$ and since the radiation energy of the Universe when all the $S$ scalars decay will simply be given by $\rho_{S}$ at this time, the decay temperature will be the same as that given in equation (41).

We must also ensure that the $S$ condensate is not thermalized by scattering processes involving the thermal background particles. We first show that the $X$ particles are always heavier than $T$ and so Boltzmann suppressed if the coupling $\lambda_{a}$ is not too small compared with 1 . The mass of the $X$ particles from the $X-N$ 
mass matrix is given by $\bar{M}_{X} \approx \operatorname{Max}\left(M_{X}, \lambda_{a}<S>\right)$. Therefore $\bar{M}_{X} \gtrsim 10^{10} \mathrm{GeV}$ if $M_{X} \gtrsim 10^{10} \mathrm{GeV}$. Since, from equation (1), $T_{r}$ at $H \approx m_{s}$ is given by

$$
T_{r}\left(H \approx m_{s}\right) \approx 3.2 \times 10^{9}\left(\frac{T_{R}}{10^{9} G e V}\right)^{1 / 2} G e V \quad(46)
$$

we see that $\bar{M}_{X} \gtrsim T$ for all $H$ less than $m_{s}$. At $H \approx m_{s}$ the $X$ mass will be given by $\bar{M}_{X} \approx \lambda_{a}<S>$ so long as $\lambda_{a} \gtrsim 2 \times 10^{-5} \lambda_{S}^{1 / 4}\left(\frac{M_{X}}{10^{10} \mathrm{GeV}}\right)$. Assuming that this is satisfied, we see that since $\left\langle S>\propto H \propto T^{4}\right.$ for $H \gtrsim m_{s}, \bar{M}_{X}$ will be larger than $T$ for all temperatures after the end of inflation. Thus the $X$ particles will not thermalize the condensate for $\lambda_{a} \gtrsim 10^{-5}$.

We next consider whether the $N$ particles can thermalize the condensate. The $N$ particle mass from the $X-N$ mass martix is given by $\bar{M}_{N} \approx \operatorname{Min}\left(\lambda_{a}<S>\right.$ ,$\left.\frac{\lambda_{a}^{2}<S>^{2}}{M_{X}}\right)$. In the following, for simplicity, we will concentrate on the case of large $T_{R}$, not much smaller than the gravitino upper bound, which is of most interest to us here. Smaller values of $T_{R}$ can be analysed in a similar way. Let $T_{N}$ be the temperature below which $\bar{M}_{N}$ becomes less than $T$. Let $T_{c}$ be the temperature

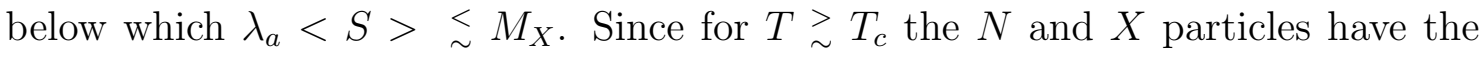
same mass and we have shown than $\bar{M}_{X} \gtrsim T$ for all $T, \bar{M}_{N}$ can only become less than $T$ at temperatures less than or equal to $T_{c}$. Assuming that $T_{c} \approx T_{R}, T_{c}$ is given by

$$
T_{c} \approx \frac{2 \times 10^{7} \lambda_{s}^{1 / 6}}{\lambda_{a}^{2 / 3}}\left(\frac{m_{s}}{100 G e V}\right)^{1 / 2}\left(\frac{M_{X}}{10^{10} G e V}\right)^{2 / 3}\left(\frac{10^{9} G e V}{T_{R}}\right)^{1 / 3} \mathrm{GeV}
$$

Therefore with $\lambda_{a}$ not too small, say in the range 0.01 to 1 , this will be less than $T_{R}$ for $M_{X} \approx 10^{10} \mathrm{GeV}$ and for reheating temperatures not much smaller than the gravitino upper bound. $\bar{M}_{N}$ can only be less than $T$ if $T_{N} \lesssim T_{c}$. In this case $T_{N}$ is given by

$$
T_{N} \approx \frac{1.2 \times 10^{5} \lambda_{S}^{1 / 4}}{\lambda_{a}}\left(\frac{m_{s}}{100 G e V}\right)^{3 / 4}\left(\frac{M_{X}}{T_{R}}\right)^{1 / 2} \mathrm{GeV}
$$

This is indeed less than $T_{c}$ if

$$
\lambda_{a}>7 \times 10^{-6} \lambda_{S}^{1 / 4}\left(\frac{m_{s}}{100 G e V}\right)^{3 / 4}\left(\frac{10^{10} G e V}{M_{X}}\right)^{1 / 2}\left(\frac{10^{9} G e V}{T_{R}}\right)^{1 / 2}
$$


This will be easily satisfied for values of $T_{R}$ not much smaller than the gravitino upper bound, $M_{X} \approx 10^{10} \mathrm{GeV}$ and $\lambda_{a}$ in the range 0.01 to 1 . Therefore in this case we must apply the no thermalization condition during radiation domination at $T_{N}$. Thermalization will primarily occur via the effective interaction of equation (36). We treat the scattering of the thermal $N$ particles from the zero momentum $S$ condensate scalars as a simple scattering process involving $S$ and $N$ particles. The scattering rate for $S \psi_{N} \leftrightarrow S \psi_{N}$ is then given by

$$
\Gamma_{s c} \approx \frac{1}{\pi^{3}} \frac{\lambda_{a}^{4} T^{3}}{M_{X}^{2}} \quad(50)
$$

Requiring that the no thermalization condition, $\Gamma_{s c} \lesssim H$, be satisfied at $T_{N}$, equation (48), then gives for the no thermalization constraint on $T_{R}$,

$$
T_{R} \gtrsim 6 \times 10^{12} \lambda_{a}^{6}\left(\frac{m_{s}}{100 G e V}\right)^{3 / 2}\left(\frac{10^{10} G e V}{M_{X}}\right)^{3} G e V \quad \text { (51). }
$$

$\lambda_{a} \lesssim 0.2$ and $M_{X} \approx 10^{10} \mathrm{GeV}$ will easily allow a range of values for $T_{R}$ below the gravitino upper bound to be consistent with no thermalization of the $S$ condensate. Thus with, for example, $\lambda_{a} \approx 0.1, M_{X} \approx 10^{10} \mathrm{GeV}$ and $T_{R} \approx 10^{7-9} \mathrm{GeV}$, the $S$ condensate will evade thermalization and will safely decay at a temperature of around $10 \mathrm{MeV}$.

We next consider the conditions under which the $S$ condensate can eliminate the NMSSM domain walls. We first consider the case where the Universe is radiation dominated. The terms in the SUSY scalar potential responsible for the energy density splitting are given by

$$
\frac{2 k \lambda_{a}^{2}}{M_{X}}\left(S^{2} N N^{\dagger 2}+\text { h.c. }\right)
$$

This is explicitly dependent on the phase of the $S$ field and has a non-zero average over time. It results in a splitting of energy density of the different $N$ vacuum states, $\Delta V$, given by

$$
\Delta V \approx \frac{k \lambda_{a}^{2}}{M_{X}}<S^{2}><N>^{3}
$$

Thus the condition for $\Delta V$ to be large enough to eliminate relativistic domain walls 
at a temperature $T$ during radiation domination, $\Delta V(T) \gtrsim \sigma H(T)$, becomes

$$
<S^{2}>>\frac{k_{T}(T) T^{2}}{k \lambda_{a}^{2}} \frac{M_{X}}{M_{P l}} \frac{m_{s}}{<N>}
$$

Since $<S^{2}>$ is proportional to $T^{3}$, we see that this is most easily satisfied as soon as the walls become relativistic at $T_{r e l}$. Thus in order to eliminate the domain walls during radiation domination we require that

$$
T_{R}>\frac{k\left(T_{r e l}\right)}{\alpha_{\rho}\left(T_{r e l}\right) k \lambda_{a}^{2}} \frac{1}{T_{r e l}} \frac{m_{s}}{<N>} \frac{m_{s}^{2} M_{X} M_{P l}}{S_{o}^{2}}
$$

For example, if we consider the domain wall to become relativistic shortly after the quark-hadron phase transition [7], say at $T_{r e l} \approx 0.1 \mathrm{GeV}$, and to be too strongly damped for the pressure due to $\Delta V$ to be able to collapse the domain walls at higher temperatures, then we obtain

$$
T_{R} \gtrsim 5 \times 10^{3} \frac{f_{c} \lambda_{s}^{1 / 2}}{k \lambda_{a}^{2}}\left(\frac{m_{s}}{<N>}\right)\left(\frac{m_{s}}{100 G e V}\right)^{3 / 2}\left(\frac{0.1 G e V}{T_{\text {rel }}}\right)\left(\frac{M_{X}}{10^{10} G e V}\right) G e V
$$

We have included a correction factor, $f_{c}$, for the case where the domain walls become relativistic during $S$ condensate matter domination rather than during radiation domination. We have shown that the Universe becomes matter dominated at a temperature $T_{d o m}$, equation (45), which is in the tens of GeVs for $T_{R}$ close to the gravitino upper bound. In this case, for $T_{r e l}<T_{d o m}$, we have to modify the domain wall constraint on $T_{R}$. Once $T \lesssim T_{d o m}$, entropy will be effectively conserved and the radiation energy density will be mostly due to the primordial radiaiton. However, there will also be additional radiation due to the decay of the $S$ condensate, with a temperature given by equation (1) with the replacement $T_{R} \rightarrow T_{d}$. This radiation will dominate the primordial radiation once $T \lesssim T_{*}$, where

$$
T_{*}=\left(\frac{4 g\left(T_{d}\right)}{25}\right)^{1 / 5} T_{d}^{4 / 5} T_{d o m}^{1 / 5}
$$

Once $T \lesssim T_{*}$ entropy is no longer effectively conserved and $H \propto T^{4}$ drops much more rapidly with $T$. The result of all this on the domain wall bound is to introduce a correction factor $f_{c}$, where, for $T_{r e l}>T_{*}$,

$$
f_{c}=\left(\frac{T_{\text {dom }}}{T_{\text {rel }}}\right)^{1 / 2}
$$


and for $T_{r e l}<T_{*}$

$$
f_{c}=\left(\frac{g\left(T_{*}\right)}{g\left(T_{r e l}\right)}\right)^{1 / 2}\left(\frac{T_{*}}{T_{r e l}}\right)^{5}\left(\frac{T_{\text {dom }}}{T_{*}}\right)^{1 / 2}
$$

The weakest lower bound on $T_{R}$ is obtained when $T_{r e l} \gtrsim T_{*}$. $T_{r e l} \geq T_{*}$ is satisfied if

$$
T_{d} \lesssim 40 \lambda_{s}^{1 / 8}\left(\frac{1}{g\left(T_{d}\right)}\right)^{1 / 4}\left(\frac{T_{r e l}}{0.1 G e V}\right)^{5 / 4}\left(\frac{100 G e V}{m_{s}}\right)^{1 / 8}\left(\frac{10^{9} G e V}{T_{R}}\right)^{1 / 4} M e V \quad(60) .
$$

The lower bound on $T_{R}$ from requiring the elimination of the domain walls then becomes

$$
T_{R} \gtrsim 5.4 \frac{\lambda_{s}^{1 / 2}}{k^{2} \lambda_{a}^{4}}\left(\frac{m_{s}}{100 G e V}\right)^{7 / 2}\left(\frac{0.1 G e V}{T_{r e l}}\right)^{3}\left(\frac{M_{X}}{10^{10} G e V}\right)^{2} G e V \quad \text { (61). }
$$

For example, this allows a range of reheating temperatures from $T_{R} \approx 5 \times 10^{6} \mathrm{GeV}$ up to the gravitino bound to be compatible with elimination of the domain walls at $T_{\text {rel }} \approx 0.1 \mathrm{GeV}$ when $\lambda_{a}$ is in the range 0.01 to $1, k \approx 0.1$ and $M_{X} \approx 10^{10} \mathrm{GeV}$.

Thus we see that, with $\lambda_{a} \approx k \approx 0.1, M_{X} \approx 10^{10} \mathrm{GeV}$ and $T_{R} \approx 10^{7-9} \mathrm{GeV}$, the $S$ condensate can evade thermalization, can safely decay before nucleosynthesis (at a temperature of around $10 \mathrm{MeV}$ ) and can eliminate the NMSSM domain walls, without requiring any small renormalizible couplings.

We should note that the preferred order of magnitude for $M_{X}$ in this model is around $10^{10} \mathrm{GeV}$. Values smaller than this make it difficult to satisfy the no thermalization constraint, equation (51), whilst values larger than this make it difficult to eliminate the domain walls, equation (61), and to ensure that the condensate decays before nucleosynthesis, equation (43).

So far we have not explained why it was necessary to introduce the second discrete symmetry $Z_{B}$. In the absence of this discrete symmetry, we could have had an additional term in the non-renormalizible superpotential,

$$
\frac{\lambda_{b}}{M_{P l}} S^{3} X \quad(62),
$$

where $\lambda_{b} \approx 1$. On integrating out the $X$ field, this would give an effective operator in the superpotential,

$$
\frac{\lambda_{a} \lambda_{b}}{M_{X} M_{P l}} S^{4} N \quad(63)
$$


which lifts the flat direction much earlier than the $S^{6}$ superpotential term, such that

$$
S_{o} \approx\left(\frac{m_{s} M_{X} M_{P l}}{\lambda_{a} \lambda_{b}}\right)^{1 / 3}
$$

As a result, the lower bound on $T_{R}$ from the requirement that the domain walls can be eliminated during radiation domination would become

$$
T_{R} \gtrsim 3 \times 10^{12} \frac{\lambda_{b}^{2 / 3}}{k \lambda_{a}^{4 / 3}}\left(\frac{m_{s}}{100 G e V}\right)^{4 / 3}\left(\frac{0.1 G e V}{T_{\text {rel }}}\right)\left(\frac{M_{X}}{10^{10} G e V}\right)^{1 / 3} \mathrm{GeV}
$$

Even with $k \approx \lambda_{a} \approx 1$ this would require that $M_{X} \lesssim 10^{2} \mathrm{GeV}$ in order to allow reheating temperatures below the gravitino bound, making it impossible to avoid thermalizing the condensate.

Thus, with non-renormalizible couplings of $S$ to the NMSSM fields, the $Z_{3}$ domain wall problem of the NMSSM can be solved even if the renormalizible couplings are large and the reheating temperature is not small compared with the gravitino upper bound. The introduction of an intermediate mass scale of the order of $10^{10} \mathrm{GeV}$ is essential for the solution of the domain wall problem in this case, since it provides non-renormalizible operators which are strong enough to enable the oscillating scalar field to decay fast enough to avoid problems with nucleosynthesis and to allow it to provide a sufficiently large pressure to eliminate the NMSSM domain walls whilst still being weak enough to prevent thermalization of the discrete symmetry breaking scalar condensate before the NMSSM domain walls have formed. 


\section{Conclusions.}

We have considered the possibility of solving the NMSSM $Z_{3}$ domain wall problem by spontaneous discrete symmetry breaking occuring during inflation. For the case of renormalizible couplings of the discrete symmetry breaking scalar $S$ to the NMSSM sector we find that, if the $S$ scalar has a negative hidden sector mass squared term, then it is possible to solve the NMSSM domain wall problem without requiring extremely small couplings so long as the reheating temperature is not very large compared with that of the electroweak phase transition. Reheating temperatures larger than $10^{7} \mathrm{GeV}$ would require the couplings to be less than $10^{-5}$. A solution with a positive hidden sector mass squared for $S$ always requires very small couplings and so seems generally unnatural.

For the case of non-renormalizible, Planck-scale suppressed operators, a negative hidden sector mass squared solution appears generally ruled out by the generation of a large SUSY mass for the $N$ field of the NMSSM. For the case of a positive hidden sector mass squared, we find that it is possible to solve the NMSSM domain wall problem via a coherently oscillating $S$ scalar without any small couplings, even if the reheating temperature after inflation is large. This requires the introduction of an intermediate mass scale of the order of $10^{10} \mathrm{GeV}$, in order to allow the $S$ scalars to decay without cosmological problems, and an additional discrete symmetry in order to control the allowed non-renormalizible terms and so avoid suppressing the density of coherent $S$ scalars.

Although we have concentrated on the $Z_{3}$-symmetric NMSSM, the coherently oscillating scalar mechanism for eliminating weak scale domain walls in SUSY models should have rather general applications to any SUSY model with a weak scale domain wall problem, for example, SUSY models with spontaneous CP violation or spontaneous R-parity violation at the $100 \mathrm{GeV}$ to $1 \mathrm{TeV}$ scale. In these cases,

given the sensitivity of the strong $\mathrm{CP}$ parameter $\bar{\theta}$ to explicit $\mathrm{CP}$ violating terms and the baryon number violation rate to explicit R-parity breaking terms, it may well be advantageous to have a discrete symmetry breaking expectation value which 
vanishes at zero temperature, as happens in the case of the coherently oscillating scalar mechanism.

This research was supported by a European Union Marie Curie Fellowship under the TMR programme, contract number ERBFMBICT 950567. 


\section{References}

[1] H.P.Nilles, Phys.Rep. 110 (1984) 1.

[2] G.F.Giudice and A.Masiero, Phys.Lett. B206 (1988) 480.

[3] H.P.Nilles, M.Srednicki and D.Wyler, Phys.Lett. 120B (1983) 346.

[4] U.Ellwanger, M. Rausch de Traubenberg and C.A.Savoy, Nucl.Phys. B492 (1997) 21.

[5] Ya.B.Zel'dovich, I.Yu.Kobzarev and L.B.Okun, Sov.Phys. JETP 40 (1975) 1, T.W.B.Kibble, J.Phys. A9 (1976) 1387.

[6] A.Vilenkin, Phys. Rep. 121 (1985) 263.

[7] S.A.Abel, S.Sarkar and P.L.White, Nucl.Phys. B454 (1995) 663.

[8] J.Bagger and E.Poppitz, Phys.Rev.Lett 71 (1993) 2380, J.Bagger, E.Poppitz and L.Randall, Nucl.Phys. B455 (1995) 59.

[9] S.A.Abel, Nucl.Phys. B480 (1996) 55.

[10] M.Dine, L.Randall and S.Thomas, Nucl.Phys. B458 (1996) 291.

[11] J.Ellis, A.Linde and D.Nanopoulos, Phys.Lett. 118B (1982) 59, M.Yu.Khlopov and A.Linde, Phys.Lett. 138B (1984) 265, J.Ellis, J.E.Kim and D.V.Nanopoulos, Phys.Lett. 145B (1984) 181.

[12] E.W.Kolb and M.S.Turner, The Early Universe, (Addison-Wesley, Reading MA $(1990))$.

[13] S.Sarkar, Rep.Prog.Phys. 59 (1996) 1493.

[14] R.K.Schaefer and Q.Shafi, Phys.Rev. D47 (1993) 1333; A.R.Liddle, ibid. D49 (1994) 739 . 
[15] E.Copeland, A.Liddle, D.Lyth, E.Stewart and D.Wands, Phys.Rev. D49 (1994) 6410 ,

E.D.Stewart, Phys.Rev.D51 (1995) 6847, M.Dine, L.Randall and S.Thomas, Phys.Rev.Lett. 75 (1995) 398.

[16] P.Binetruy and G.Dvali, Phys.Lett. 388B (1996) 241, E.Halyo, Phys.Lett. 387B (1996) 43.

[17] L.Dolan and R.Jackiw, Phys.Rev. D9 (1974) 3320.

[18] K.Enqvist, A.Riotto and I.Vilja, OUTP-97-49-P, hep-ph/9710373.

[19] I.A.Affleck and M.Dine, Nucl.Phys. B249 (1985) 361.

[20] J.McDonald, Phys.Lett. 357B (1995) 19.

[21] M.Dine, R.G.Leigh, P.Huet, A.Linde and D.Linde, Phys.Rev. D46 (1992) 550.

[22] J.Ellis, D.V.Nanopoulos and D.Seckel, Nucl.Phys. B259 (1985) 175.

[23] M.H.Reno and D.Seckel, Phys.Rev. D37 (1988) 3441. 